\title{
Optimal Placement of Shunt Capacitor in Distribution System by Particle Swarm Optimization
}

\author{
Shahzor Ahmed ${ }^{* 1}$, Mahesh Kumar ${ }^{1}$, Amir Mahmood Soomro ${ }^{1}$ \\ ${ }^{1}$ Institute of Information and Communication Technologies, \\ Mehran University of Engineering and Technology Jamshoro, Sindh PAKISTAN \\ *Corresponding author \\ DOI: https://doi.org/10.30880/ijie.2020.12.06.029 \\ Received 20 February 2020; Accepted 20 July 2020; Available online 30 July 2020
}

\begin{abstract}
The distribution system in power system have high resistance to reactance ratio therefore it provides more power losses and voltage drop issues. Furthermore, the load used in distribution side is mostly of inductive in nature and gives reactive power losses. Therefore, an attempt is made to integrate shunt capacitor banks to improve reactive power losses and to maintain the voltage profile in the allowable limit. Moreover, the proposed model is tested on $11 \mathrm{kV}$ Hyderabad Electric Supply Corporation (HESCO) distribution feeder for analysis of reactive power. An artificial intelligence technique named as particle swarm optimization (PSO) is used to identify the optimal placement of shunt capacitor. The proposed method reduces more than $40 \%$ power losses and gives better results in voltage profile (i.e. more than 0.95 p.u.) at all buses.
\end{abstract}

Keywords: Shunt Capacitor, HESCO, reactive power loss, artificial intelligence technique, distribution system

\section{Introduction}

Shunt capacitors are used to compensate the reactive power at the distribution end. Shunt capacitors bank are mostly used to reduce the reactive power loss and to improve the voltage profile in the system [1]. The use of SCBs is increased because they are relatively cheap, easy and quick to install and can be installed virtually anywhere in the network. Therefore, it is important to obtain the optimal size and location of capacitor. During last years, several algorithms and techniques are introduced for locating the shunt capacitor at optimal location. $\mathrm{Ng}$ et al [2] shunt capacitors can be placed into system through four different techniques types. That are numerical programming, analytical, artificial intelligence and heuristic technique. duran [3] published a report that find the shunt capacitor size as discreate variable using dynamic programming. S.gopiya [4] et al performed the analytical method for optimum allocation of shunt capacitor in the distribution system to minimize total active power losses, summarized that the capacitors have improved the voltage profile. . Sayyad et al [5] used the new mixed nonlinear programming method for capacitor situation in network and compared mathematical outcomes with alternative strategies. For last two decades artificial intelligence techniques are widely used to find the optimal size and location of capacitor. Sarfraz et al [6] proposed a technique for determining the size and location of shunt capacitor, used PVSC for determining the location and size of shunt capacitor. The results obtained were associated with previous practices. Adel et al [7] a method based on two stage technique LSI and ACO algorithm was used to locate the optimal location and size of capacitor of capacitor for power loss and voltage profile improvement. E.S. Ali et al improved harmony algorithum to create a function that could minimize overall cost and losses. George et al [8] used ant lion optimizer (ALO) to decide optima Var capacity and location of the fixed capacitor. Objective of this research was to diminish the total power losses as well as total annual cost on the distribution side. V.Tamilselvan 
et al [9] suggest at technique for optimum capacitor placement known as flower pollination algorithm. Outcomes were associated with other analytical approaches. Anwar Ali sahito at el [10] reduce the active and reactive power losses in utility system with the help of shunt capacitor as well as enhance the voltage profile, placement of capacitor was done through analytical approach and the outcome where compared with the system condition and it was concluded that the power loss reduced from $20.68 \%$ to $12.90 \%$. kennedy and russel eberhant [11] developed a technique known as particle swarm optimization. It mainly focuses on metaphor of social interaction, space for moving points in multi-dimensional space is found by adjusting the trajectories. Although, many researchers have worked on shunt capacitor and explained its benefits, but the impact of shunt capacitor varies with varying size and location of parallel capacitor in the system. Hence this work is based on optimum size and location of shunt capacitor within real time HESCO radial distribution feeder. Compensation in reactive losses and improvement in the voltage profile are the objective function for this study.

\section{Photovoltaic Process}

\subsection{Data Analysis and Network Modelling}

It is a real time radial distribution feeder of one of the Pakistan's distribution company i.e. Hyderabad electric supply company (HESCO). The feeder is named as Sarfraz Baba feeder. The network is compares of 50 busses. The feeder has standard voltage level of $11 \mathrm{KV}$. It lies at a longitude of 68.37 , latitude of 25.40 and $38 \mathrm{M}$ above the sea level as shown in Fig. 1.

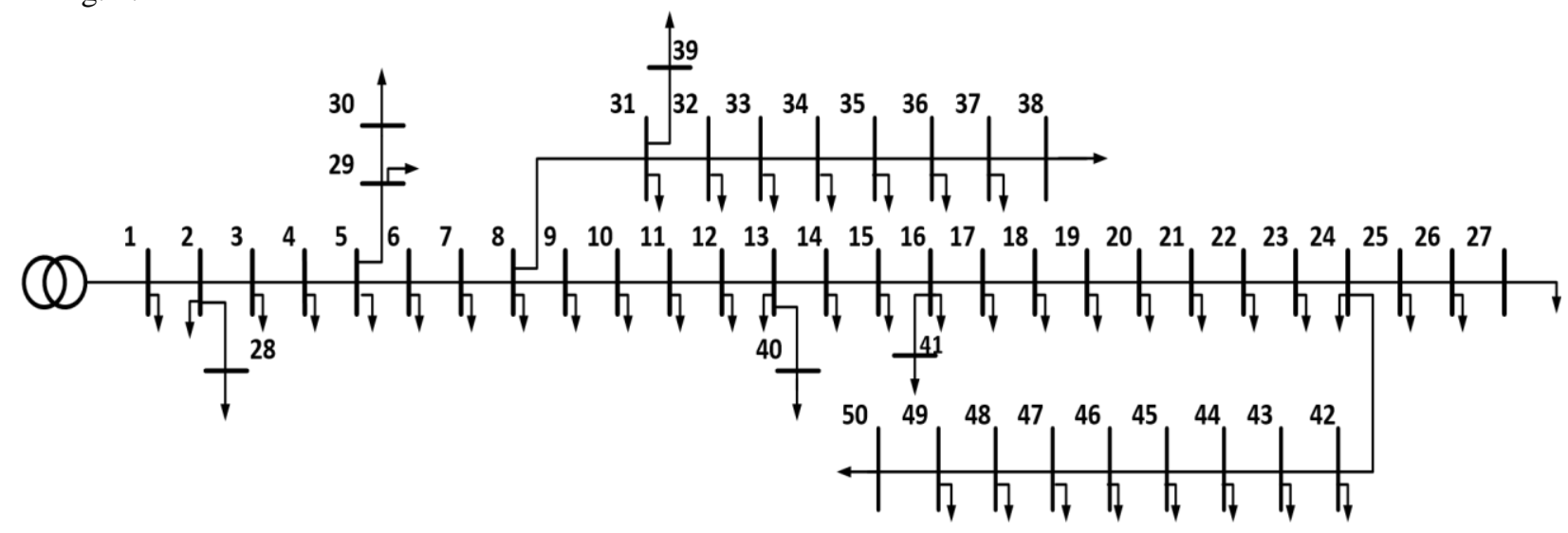

Fig. 1 - online diagram of the Sarfraz Baba Feeder HESCO

\subsection{Problem Statement}

Generally, purpose of this paper is to diminish reactive power loss component in the real time radial power distribution network. Voltage limit is set as the limitation for the system that needs to be satisfied for the quality and security purpose.

Mathematical expression for objective function is described as:

$$
f=\min \sum Q \text { loss }
$$

Where $\mathrm{f}$ is fitness function and Qloss is reactive power loss at different nodes.

\section{Placement of shunt capacitor}

As Bus-1 is assumed as slack bus so it cannot be considered for shunt capacitor therefore the upper and lower bound for shunt capacitor is as follows

\section{$2 \leq$ position $\leq$ nbuses}

\section{Voltage on load bus}

$\mathrm{V}_{\min } \leq \mathrm{V} \leq \mathrm{V}_{\max }$

\subsection{Load Flow Analysis}

Variety of load flow methods have been used by different authors in different type of application ranging from industrial automation, bio-medical industries to the power electronic applications and many others. Traditional techniques 
were previously utilized in electrical power transmission system, but distribution system is not being broadly exploited for load analysis. Distribution system possess high R/X ration and radial network topology. In this research work, 'BackwardForward sweep method', is utilized for load analysis of a real time electrical power distribution system. This method is based on a bidirectional process i.e. forward and backward. Let's consider a two-bus system starting at bus $\mathrm{K}$ and ending at bus $\mathrm{M}$ as illustrated in Fig. 2.

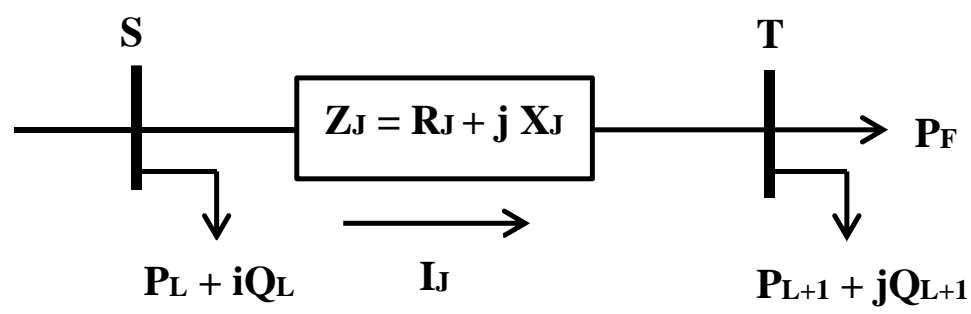

Fig. 2 - Two bus radial distribution system

At node $\mathrm{N}$, voltage is determined by

$$
V_{T}=V_{S}-I_{L} \times\left(R_{S}+j X_{S}\right)
$$

current at line is determined by

$$
I_{L}=\left(\frac{P_{L}+Q_{L}}{V_{L}}\right)
$$

Power loss is determined with Eq. (1) and (2)

$$
\begin{aligned}
& P_{L \text { loss }}=\left(\frac{P_{L+1}^{2}+Q_{L+1}^{2}}{\left|V_{L+1}^{2}\right|}\right) \times R_{L} \\
& Q_{L \text { loss }}=\left(\frac{P_{L+1}^{2}+Q_{L+1}^{2}}{\left|V_{L}^{2}\right|}\right) \times X_{L}
\end{aligned}
$$

The objective function of this work is to minimize the reactive power loss and is denoted by Eq (7)

$$
T_{\text {loss }}=\min \left(\sum_{L=1}^{n \text { bus }-1} Q_{L \text { loss }}\right)
$$

\section{Methodology}

An artificial intelligence based Particle Swarm Optimization (PSO) method is used which an evolutionary optimization algorithm introduced by Dr. Eberhart and Dr. Kennedy in 1995. This algorithm is an was encouraged by the grouping of bird in search of food. The technique is being broadly used in various fields of science and engineering due to its effective problem-solving capability [13]. The stochastics evolutionary algorithm consists group of ' $n$ ' number of particles and fitness function's possible solution is denoted by each particle's position in ' $\mathrm{D}$ ' dimensional space. Particle's velocity and position can be update with the help of following equations and the process continues till the criteria for stopping is met.

$$
\mathrm{V}_{\mathrm{S}, \mathrm{T}}^{\text {new }}=\omega . \mathrm{V}_{\mathrm{S}, \mathrm{T}}^{\text {old }}+\operatorname{randn} \mathrm{k} \times \alpha+\left(\mathrm{P}_{\mathrm{S}, \mathrm{T}}^{\text {global best }}-\mathrm{P}_{\mathrm{S}, \mathrm{T}}^{\text {old }}\right) \times \beta
$$

Where $\alpha$ and $\beta$ shows the acceleration constants and value of randn varies from 0 to 4 If outcome is not obtained then location is upgraded by Eq. (9)

$$
\mathrm{P}_{\mathrm{S}, \mathrm{T}}^{\text {new }}=\mathrm{P}_{\mathrm{S}, \mathrm{T}}^{\text {old }}+\mathrm{V}_{\mathrm{S}, \mathrm{T}}^{\text {new }}
$$

Where $\mathrm{T}=1,2,3 \ldots, \mathrm{z}$ and $\mathrm{S}=1,2,3 \ldots, \mathrm{y}$

To obtain the accurate location it is necessary to diminish the error with the repetition of process as expressed by Eq (10)

$$
\mathrm{P}_{\mathrm{S}, \mathrm{T}}^{\text {new }}=(1-\beta) \times \mathrm{P}_{\mathrm{S}, \mathrm{T}}^{\mathrm{old}}+\beta \times \mathrm{P}_{\mathrm{S}, \mathrm{T}}^{\text {global best }}+\alpha \times \text { randn } \mathrm{k}
$$




\section{A. Implementation of PSO}

The optimum size and location for shunt capacitor is identified using PSO. Table describes the basic structure of the optimization technique

Step 1 find the shunt capacitor location

- Insert the distribution system branch impedance values and power data

- Determine the sensitivity factors

- Select the buses having great number of sensitivity factor as capacitor positions

Step 2 Optimization by particle swarm algorithm

- Insert PSO data

- $\quad$ begin population with random string

- Check voltage constraints

- Obtain the power losses

Step 3 Compute the former best performance of each particle and save it as pbest

Step 4 Compute the former best performance of all particle and save it as gbest

Step 5 Compute the velocity of each particle using pbest and gbest

Step 6 Update each particle

Step 7 If founded particle satisfies all constraints and is improved then pervious standards then change the pbest new standard

Step 8 Analyze the best values of all pbest and save as gbest

Step 9 The attained value is gbest, the global solution attained by algorithm interpret it and find the capacitor values at different situations

Moreover, the optimized shunt capacitor siting and sizing in radial distribution network involves multiple steps that are requisite. Moreover, Fig. 3 shows the compete flow chart for the implementation of proposed model. 


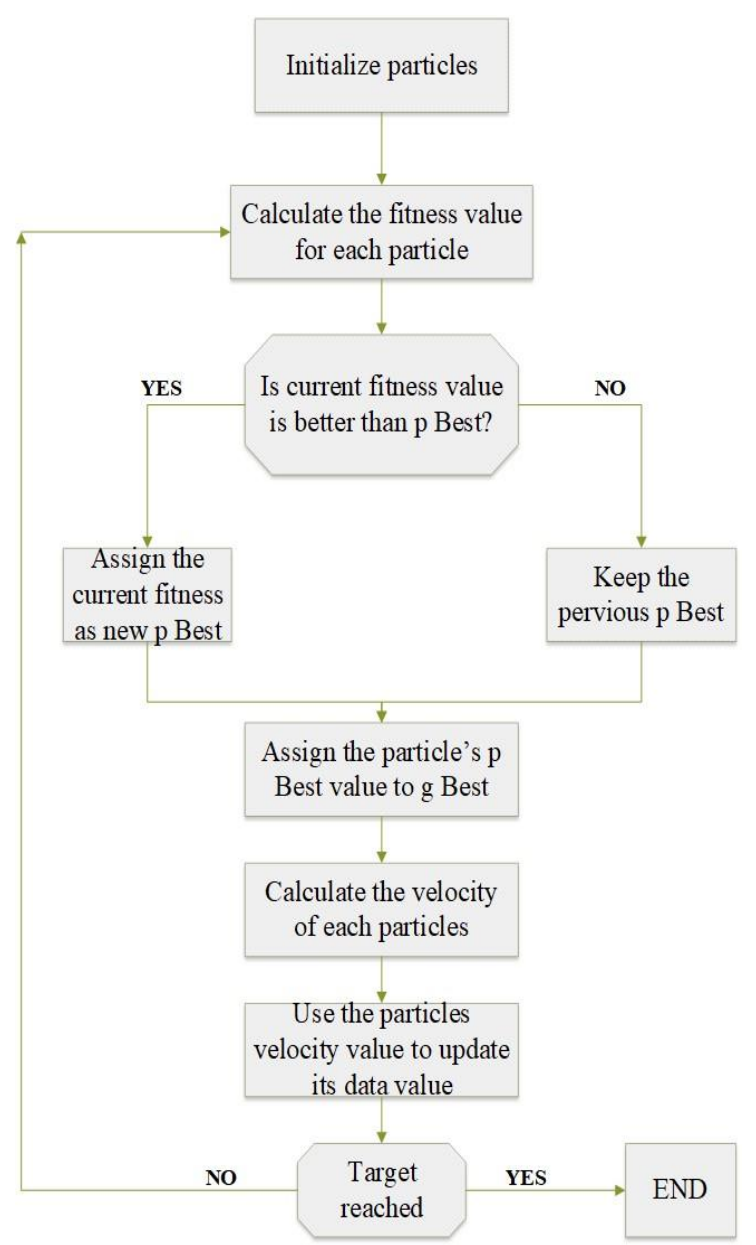

Fig. 3 - Flow chart of PSO implementation in order to obtain optimized results of the system

\section{Results and Discussion}

This study is accomplished for a real time $11 \mathrm{kV}$ radial electrical power distribution system named as Sarfaraz Baba, to observe the power loss reduction using optimized sizing and allocation of capacitor. Feeder consists of 50 buses. The purpose for this research work is to improve voltage profile and reeducation in reactive power loss. Shunt capacitors is considered as cost effective source to be installed in order to compensate the loss occurring in distribution network. Upper and lower bound of voltage for optimization are set as 1.05 and 0.95 respectively. Bus-1 in the network is assumed as slack bus. Slack bus voltage is selected as $1.02 \mathrm{~V}$ and MVA is standard as $11 \mathrm{k}$. The voltage profile value after placement of one capacitor and two capacitor are shown in Fig. 4.

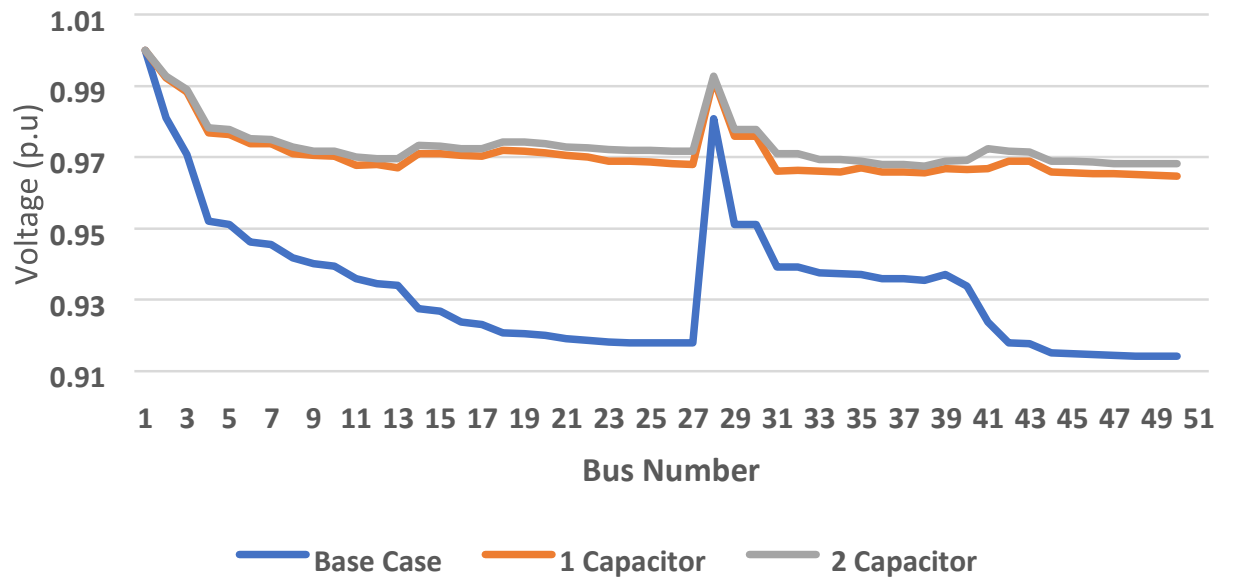

Fig. 4 - voltage profile of the system

The graph visibly clarifies that the shunt capacitor installation affects the network positively and the bus voltage is enhanced on nearly every bus. The voltage profile improvement with shunt capacitors is given below in Table 1 . 
Table 1 - Minimum voltage profile values before and after capacitor placement

\begin{tabular}{|l|l|l|l|l|l|}
\hline \multicolumn{9}{|c|}{ Voltage (P.U) } \\
\hline \multicolumn{3}{|c|}{ Base case } & After placement of one capacitor & \multicolumn{2}{|c|}{ After placement of two capacitor } \\
\hline minimum & maximum & minimum & maximum & minimum & maximum \\
\hline $0.9142 @$ bus 50 & 1.0 @ bus 01 & 0.9647 @ bus 50 & 1.0 bus 01 & $0.9682 @$ bus 50 & 1.0 bus 01 \\
\hline
\end{tabular}

As shown above the minimum system voltage before installation of capacitor is found to be 0.914 p.u. whereas the installation of one shunt capacitor improves the voltage to 0.9647 p.u, and with two capacitor installation voltage further improves up to 0.9682 p.u. The active and reactive power loss for one and two capacitors are shown in Fig. 5 and Fig. 6.

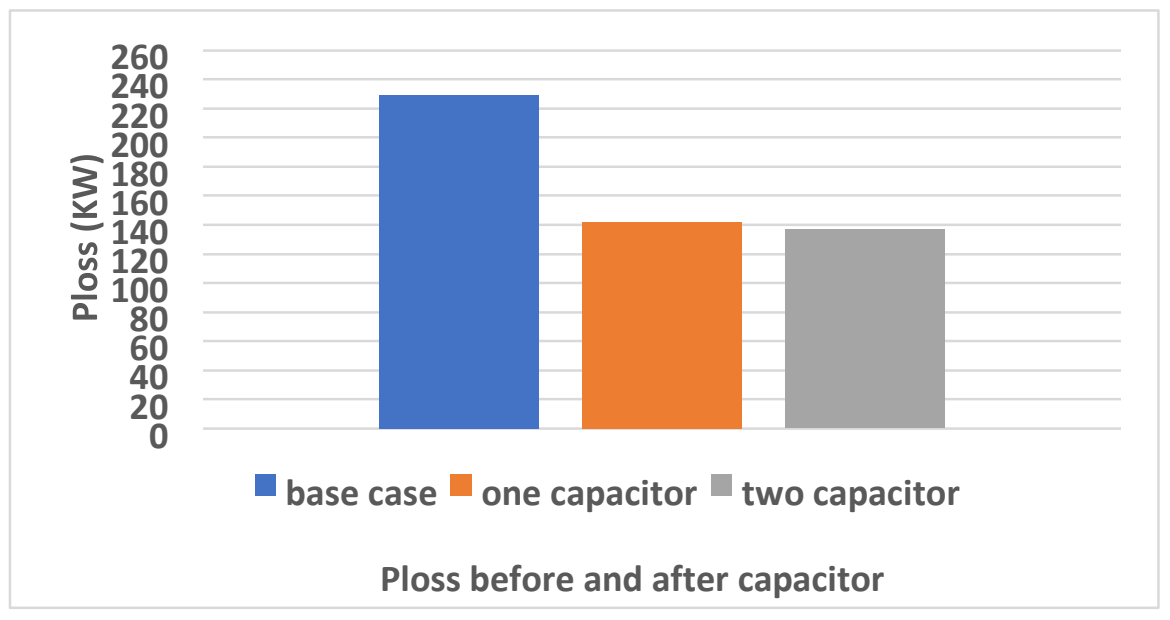

Fig. 5 - comparative analysis of active power loss of the network

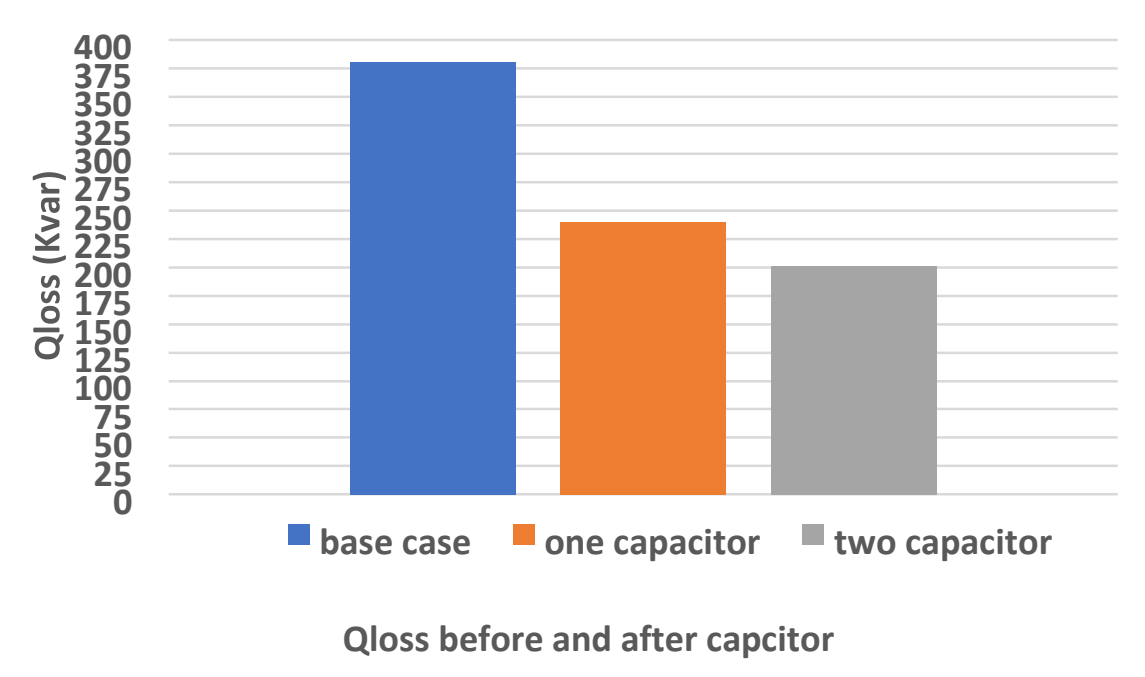

Fig. 6 - comparative analysis of reactive loss of the network

From the figure above the improvement of active power and considerable decrease in reactive power requirements it is also shown below in Table 2 and Table 3. 
Table 2 - Active and reactive power losses for one capacitor before and after capacitor placement

\begin{tabular}{|l|l|l|l|l|l|}
\hline \multicolumn{2}{|c|}{ Before capacitor } & \multicolumn{2}{c|}{ with one capacitor } & \multicolumn{2}{c|}{ Loss 'eduction } \\
\hline Real power & Reactive power & Real power & Reactive power & real & reactive \\
\hline $228.9 \mathrm{~kW}$ & $380.6 \mathrm{kVar}$ & $142.1 \mathrm{~kW}$ & $239.9 \mathrm{kVar}$ & $37.92 \%$ & $36.77 \%$ \\
\hline
\end{tabular}

Table 3 - Active and reactive power losses for one capacitor before and after capacitor placement

\begin{tabular}{|l|l|l|l|l|l|}
\hline \multicolumn{2}{|c|}{ Before capacitor } & \multicolumn{2}{c|}{ With two capacitors } & \multicolumn{2}{c|}{ Loss reduction } \\
\hline Real power & Reactive power & Real power & Reactive power & Real & reactive \\
\hline $228.9 \mathrm{~kW}$ & $380.6 \mathrm{kVar}$ & $137.1 \mathrm{~kW}$ & $201.2 \mathrm{kVar}$ & $40.26 \%$ & $47.13 \%$ \\
\hline
\end{tabular}

\subsection{Convergence of PSO Algorithm}

PSO is one of the most widely used algorithm in distribution system and its higher efficiency with fast convergence. Figure represents the convergence curve for the $11 \mathrm{kV}$ Sarfaraz Baba Feeder. Above simulation results suggests that PSO, in comparison with other AI technique has faster convergence i.e. converges within a few iterations as shown in Fig. 7.

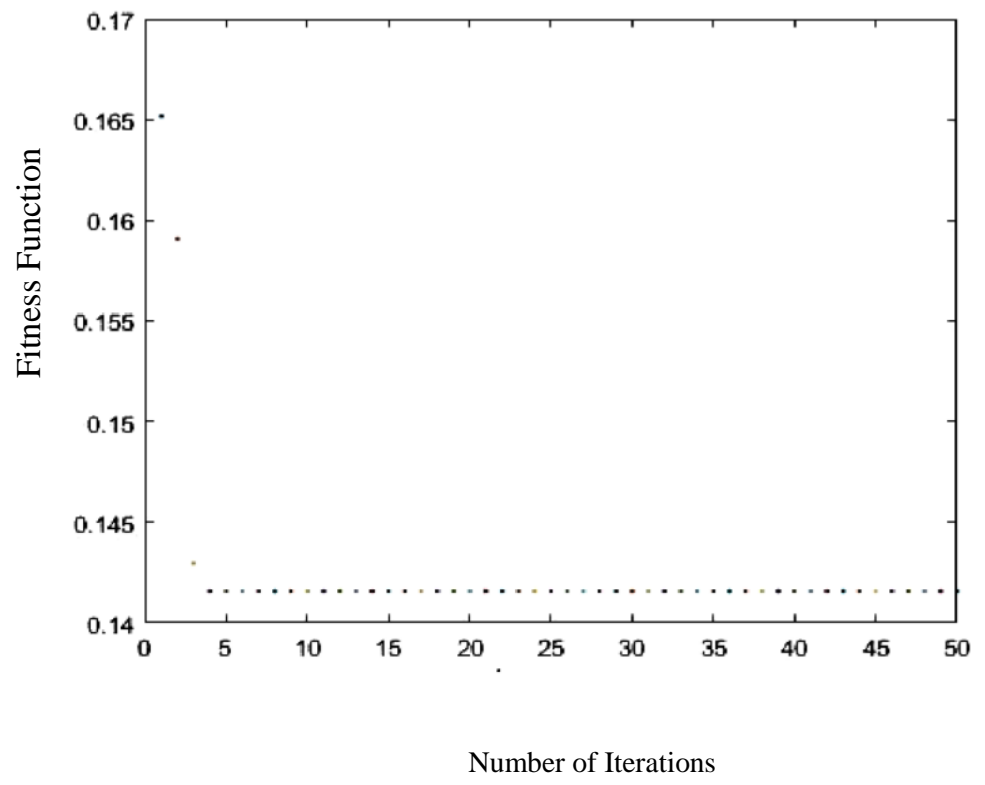

Fig. 7 - convergence of PSO

\section{Conclusion}

This paper presented a new technique to tackle reactive power loss problem with real time networks in Pakistan. PSO is artificial intelligence technique used to determine the optimum allocation of parallel capacitor in a radial distribution network. The objective of the research is to minimize reactive power loss and improve in voltage profile of $11 \mathrm{kV} \mathrm{HESCO}$ radial feeder. Results suggest that optimum size and location plays an important role for reactive losses reduction. The optimal location and size of shunt capacitor is at 19th bus of $2.460 \mathrm{k}$ Var. The active power loss was $228.9 \mathrm{k} \mathrm{W}$ and it reduced to $142.1 \mathrm{~kW}$ i-e. $37.92 \%$ and considerable reduction in reactive power loss of system was observed and it was reduced from $380.6 \mathrm{k}$ Var to $239.9 \mathrm{kVar}$ showing $36.77 \%$ in the system. The optimal location and size of two shunt capacitor is 15 th and 44 th bus of 2.278 and $1.050 \mathrm{k}$ Var. The active power loss was $228.9 \mathrm{k} \mathrm{W}$ and it reduced to $137.1 \mathrm{~kW}$ i-e. $40.26 \%$ and considerable reduction in reactive power loss of system was observed and it was reduced from $380.6 \mathrm{kVar}$ to $201.2 \mathrm{kV}$ ar showing $47.13 \%$ in the system. Furthermore, simulation results show that the suggested algorithm has fast convergence as compared to other methods. Likewise, results also validate effectiveness of the method for reducing apparent power loss and improvement in the voltage profile.

\section{Acknowledgement}

Researchers are gratified to MUET Jamshoro, Pakistan for untried efforts and non-ending support to accomplish this work. 


\section{References}

[1] C.F. Chang. (2008). Reconfiguration and capacitor placement for loss reduction of distribution systems by ant colony search algorithm. IEEE Trans. Power Syst., 23, 1747-1755

[2] H. N. Ng, M. M. A. Salama, and a. Y. Chikhani. (2000). Classification of capacitor allocation techniques. IEEE Trans. Power Deliv., 15, 387-392

[3] Dura, Hernando. (1968). Optimum number, location, and size of shunt capacitors in radial distribution feeders a dynamic programming approach. IEEE Transactions on Power Apparatus and Systems, 9, 1769-1774

[4] Naik, S. Gopiya, D. K. Khatod, and M. P. Sharma. (2013). Optimal allocation of combined DG and capacitor for real power loss minimization in distribution networks. International Journal of Electrical Power \& Energy Systems, 53, 967-973

[5] Nojavan, Sayyad, Mehdi Jalali, and Kazem Zare. (2014). Optimal allocation of capacitors in radial/mesh distribution systems using mixed integer nonlinear programming approach. Electric Power Systems Research, 107, 119-124

[6] Nawaz, Sarfaraz, Anajli Jain, and Abhishek Gupta. (2017). A novel technique for optimal allocation of capacitors in radial distribution system. Power, Control \& Embedded Systems (ICPCES), 2017 4th International Conference on. IEEE

[7] El-Ela, Adel Ali Abou, et al. (2016). Optimal capacitor placement in distribution systems for power loss reduction and voltage profile improvement. IET Generation, Transmission \& Distribution, 10, 1209-1221

[8] George, Thomas, et al. (2018). Ant lion optimization technique for optimal capacitor placement based on total cost and power loss minimization. Innovative Trends in Computer Engineering (ITCE), 2018 International Conference on. IEEE

[9] Tamilselvan, V., et al. (2018). Optimal capacitor placement in radial distribution systems using flower pollination algorithm. Alexandria engineering journal. 57, 2775-2786

[10] Kumar, S., ur Rehman, C. K. F., Shaikh, S. A., \& Sahito, A. A. (2018). Voltage improvement and power loss reduction through capacitors in utility network. In 2018 International Conference on Computing, Mathematics and Engineering Technologies (iCoMET). IEEE

[11] Kennedy, J. (2010). Particle swarm optimization. Encyclopedia of machine learning, 760-766 